\title{
Generalized near horizon extreme binary black hole geometry
}

\author{
Jacob Ciafre, ${ }^{1, *}$ Shahar Hadar, ${ }^{2,4, \dagger}$ Erin Rickenbach, ${ }^{1, \$}$ and Maria J. Rodriguez ${ }^{1,2,3,8}$ \\ ${ }^{1}$ Department of Physics, Utah State University, 4415 Old Main Hill Road, Utah 84322, USA \\ ${ }^{2}$ Max Planck for Gravitational Physics-Albert Einstein Institute, Am Muhlenberg 1, \\ Potsdam 14476, Germany \\ ${ }^{3}$ Instituto de Física Teórica IFT-UAM/CSIC, C/ Nicolás Cabrera 13-15, \\ Universidad Autónoma de Madrid, 28049 Madrid, Spain \\ ${ }^{4}$ Center for the Fundamental Laws of Nature, Harvard University, Cambridge, Massachusetts 02138, USA
}

(Received 27 June 2019; published 15 August 2019)

\begin{abstract}
We present a new vacuum solution of Einstein's equations describing the near horizon region of two neutral, extreme (zero-temperature), corotating, nonidentical Kerr black holes. The metric is stationary, asymptotically near horizon extremal Kerr (NHEK), and contains a localized massless strut along the symmetry axis between the black holes. In the deep infrared, it flows to two separate throats which we call "pierced-NHEK" geometries: each throat is NHEK pierced by a conical singularity. We find that in spite of the presence of the strut for the pierced-NHEK geometries the isometry group $\operatorname{SL}(2, \mathrm{R}) \times \mathrm{U}(1)$ is restored. We find the physical parameters and entropy.
\end{abstract}

DOI: 10.1103/PhysRevD.100.044033

\section{INTRODUCTION}

Rapidly rotating, (near-)extreme Kerr black holes (BHs) constitute a unique arena which offers both observational relevance and enhanced theoretical control. Several highspin candidates (cf. [1-4]) have been observed, and such $\mathrm{BHs}$ could produce characteristic signatures for various current and future experiments, including gravitationalwave detectors such as LIGO/Virgo, and optical observatories such as the recently triumphant [5] Event Horizon Telescope. Theoretically, (near-)extreme BHs are especially tractable since they develop an emergent conformal symmetry. More precisely, they admit a nondegenerate near-horizon geometry, the so-called near-horizon extreme Kerr (NHEK) geometry [6]. This geometry is interesting: every fixed polar angle slice of it can be thought of either as 2-dimensional anti-de Sitter space $\left(\mathrm{AdS}_{2}\right)$ with a circle nontrivially fibered upon it or (equivalently) as a quotient of the so-called warped $\mathrm{AdS}_{3}$ spacetime. Consequently it enhances the isometry group of Kerr, $\mathrm{R} \times \mathrm{U}(1)$ (corresponding to stationarity and axisymmetry), to $\operatorname{SL}(2, R) \times$ $\mathrm{U}(1)$. This motivated the Kerr/CFT conjecture [7], which

\footnotetext{
*jakeciafre@outlook.com

shaharhadar@g.harvard.edu

*rickenbacherin@gmail.com

\$maria.rodriguez@aei.mpg.de; maria.rodriguez@usu.edu
}

Published by the American Physical Society under the terms of the Creative Commons Attribution 4.0 International license. Further distribution of this work must maintain attribution to the author(s) and the published article's title, journal citation, and DOI. Funded by SCOAP. hypothesizes that the Kerr $\mathrm{BH}$ is dual to a $(1+1$ dimensional) conformal field theory (CFT) living on the boundary of this near horizon geometry. This boundary can be thought of as the spacetime region in which the NHEK geometry is glued to the external, asymptotically flat, Kerr spacetime.

The NHEK geometry has a simpler cousin-the Robinson-Bertotti universe or $\mathrm{AdS}_{2} \times \mathrm{S}^{2}$. This spacetime arises as an analogous near-horizon limit of maximally charged Reissner-Nordström BHs. This type of BHs can be used to construct, remarkably simply, multi-BH configurations [8]. Those are static solutions to Einstein-Maxwell theory with an arbitrary number of maximally charged (all with the same sign), nonrotating BHs of any mass. The time-independence of these solutions is possible since the BHs' gravitational attraction and electric repulsion cancel each other precisely - in the full nonlinear theory-for arbitrary $\mathrm{BH}$ positions. A neat observation regarding these solutions was made in [9]. Consider a system of two such maximally charged BHs. When they are widely separated, there exist also well-separated near-horizon (approximately $\mathrm{AdS}_{2} \times \mathrm{S}^{2}$ ) throats surrounding each one of the BHs. When the BHs are close to each other (relative to a length scale defined by a characteristic mass), however, there exists a region around them which is approximately an $\mathrm{AdS}_{2} \times \mathrm{S}^{2}$ throat which surrounds both horizons, and only when moving further towards either one of the horizons does one recover the two separate throats. This phenomenon was coined in [9] "AdS fragmentation": the joint throat fragments into two smaller ones, when moving deeper into the infrared. This generalizes to an arbitrary number of throats: 
one "trunk" throat can fragment into several branches which can then branch again, and so forth.

This compelling picture depends strongly on the properties of the special system of choice. The fact that it can be embedded in a supersymmetric theory, as a solution which preserves some supersymmetry [10], guarantees this type of behavior. In this paper, we propose the closest possible analogue, presumably, to fragmentation in the case of maximally rotating, uncharged BHs. Since these are not supersymmetric anymore and there is no known smooth stationary solution involving such BHs, we allow for a conical singularity between the BHs which balances the gravitational attraction and keeps the system stationary. We study a 1-parameter family of exact axis-symetric solutions describing two corotating extreme Kerr BHs of arbitrary masses which are held apart by a conical singularity with effective pressure, usually called a strut and as we rescale coordinates to zoom-in on the near-horizon region, we also shorten the strut separating the BHs. In this way we construct the exact solution corresponding to the region where NHEK fragments into two NHEK-like throats which are held apart by the strut. We call these "NHEK2" geometries. The solution presented here generalizes [11], which studied a similar construction for the equal-mass case. These infrared near-horizon geometries which the strut pierces on its way to the horizons are analogues of NHEK which include a conical singularity at one of the poles, extending from the horizon all the way to the NHEK boundary. We verify that this does not ruin the symmetry structure: the "pierced-NHEK" geometry still has an $\operatorname{SL}(2, R) \times U(1)$ isometry group. So while the full NHEK2 does not have $\operatorname{SL}(2, \mathrm{R}) \times \mathrm{U}(1)$, it interpolates from a geometry that does have conformal symmetry in the ultraviolet to two throats that are also conformally symmetric, in the infrared.

Introducing conical singularities has caveats which are important to stress. First, the stability, both classical and quantum mechanical, of these solutions is questionable. A second point is that the type of conical singularities we use here, the struts, are of excess angle type (rather than deficit angle); the effective stress-energy associated to such objects has negative energy density. Keeping these caveats in mind, we still hope that this construction may be useful in various contexts. First, such stationary BH binary solutions have been recently applied to study astrophysically motivated problems involving dynamical binaries (see for example [12] for the use of quasistationary, extremally charged solutions in a gravitational-wave application); even though the physics governing the dynamics of these systems is different it was argued in [13] (see also references therein) that in some cases such solutions can be used as tools for modeling the astrophysical systems' observational signatures, e.g., gravitational lensing. And secondly, these solutions may give some insight in the holographic, Kerr/CFT context. In this regard, it is interesting to note a recent study of holography and thermodynamics with conical singularities in the bulk
[14]. It should be possible to generalize our construction to an arbitrary number of BHs with arbitrary masses.

The workhorses of this paper are the binary $\mathrm{BH}$ solutions first found in [15] and further studied, including their construction via various solution generating techniques in [16-21]. These exact solutions are stationary, axisymmetric, asymptotically flat solutions which describe two rotating BHs held apart by a strut along the symmetry axis. The BHs of these solutions can have arbitrary masses and spins and in particular can be either co- or counterrotating. We are interested in the case in which the BHs are maximally corotating, with arbitrary masses. In particular, we start from the corotating solution described in [22], and for the convenience of the interested reader we describe it explicitly in the so-called Weyl-coordinates in Appendix A. This coordinate choice serves best to describe classes of stationary and axisymmetric solutions of Einstein's theory of general relativity in vacuum.

The rest of this paper is organized as follows. We first construct the new generalized near horizon geometry of the stationary binary extreme-Kerr BH solution in Sec. II and analyze its physical properties. In particular, we show how it admits a localized strut along the symmetry axis between the black holes but is asymptotically NHEK. In Sec. III we zoomin further to the infrared of each throat, and find the nearhorizon geometries in which the strut pierces the horizons, extending from the horizon all the way to the NHEK boundary. We show that in spite of the strut, the pierced-NHEK geometries have an $\mathrm{SL}(2, \mathrm{R}) \times \mathrm{U}(1)$ isometry group. Finally, we will summarize the key results of the paper in Sec. IV.

\section{GENERALIZED-NHEK2: GENERALIZED NEAR HORIZON GEOMETRY OF EXTREME BINARY KERR BLACK HOLES SOLUTION}

In this section, we construct the generalized near horizon geometry of extreme binary Kerr (Generalized-NHEK2) black hole solution. Our starting point, is the stationary solution to Einstein equations in vacuum [22] that contains two extremal (zero-temperature) corotating black holes. For convenience and for fixing the notation, we reproduced the original results of [22] in Appendix A. We will only consider the solutions characterized by positive values of the mass that correspond to the parameter range

$$
-\frac{1}{\sqrt{2}} \leq p<0, \quad q>0, \quad q<P \leq 1 .
$$

Note that for $P=+1$ the equal mass case, treated in $[11,21]$, is recovered; ${ }^{1}$ the extreme mass ratio limit is recovered for $P \rightarrow\left(\sqrt{1-p^{2}}\right)_{+}$or $P \rightarrow(-p)_{-}$.

\footnotetext{
${ }^{1}$ As described in [22], there is another solution with positive mass that corresponds to $-1<P<-p$. This solution belongs to a more problematic case containing a massless ring singularity outside the symmetry axis that we will not consider here.
} 


\section{A. Near-horizon limiting procedure}

In previous works [11] we developed the necessary tools to inspect the extreme corotating binary Kerr black hole solution. This section is nevertheless self-contained. We proceed to compute the near horizon geometry of extremal nonidentical binary Kerr black holes solution, that we are going to refer to as "generalized-NHEK2".

The solution of extremal BBHs [22]-that we reproduced in Appendix A-has a rather more compact representation in Weyl coordinates. We therefore perform the scaling computations in these coordinates. In this case, we find that the appropriate near-horizon limiting procedure for the extremal BBHs is

$$
\begin{gathered}
\rho=\hat{\rho} \lambda, \quad z=\hat{z} \lambda, \quad t=\frac{\hat{t}}{\lambda}, \quad \phi=\hat{\phi}+\frac{1}{2 M} \frac{\hat{t}}{\lambda}, \\
p=-\frac{1}{\sqrt{2}}+\frac{3 \sqrt{2}-2 P}{4} \lambda, \quad \kappa=M \lambda .
\end{gathered}
$$

Taking $\lambda \rightarrow 0$ and keeping $(\hat{t}, \hat{\rho}, \hat{z}, \hat{\phi})$ fixed. As a result of this procedure, we find the generalized (nonidentical mass) generalized-NHEK2 geometry

$d s^{2}=-\frac{\hat{\rho}^{2}}{f} d \hat{t}^{2}+f(d \hat{\phi}+\omega d \hat{t})^{2}+e^{2 \nu}\left(d \hat{\rho}^{2}+d \hat{z}^{2}\right)$,

defined by the equations

$$
\begin{aligned}
f & =-\frac{4 M^{2} \mu_{0}\left(\mu_{0}+2 \sigma_{0}^{2}\right)}{\mu_{0}\left(\mu_{0}+2 \sigma_{0}^{2}-2 \sigma_{1}+\pi_{0}\right)+\mu_{1} \pi_{1}+\left(1-y^{2}\right) \sigma_{0} \tau_{0}}, \\
\omega & =-\frac{\pi_{0} \sigma_{0}+\pi_{1} \sigma_{1}-\mu_{1}-4 \sigma_{0} \sigma_{1}-\left(1-y^{2}\right) \tau_{0} / 2}{2 M\left(\mu_{0}+2 \sigma_{0}^{2}\right)}, \\
e^{2 \nu} & =\frac{\mu_{0}\left(\mu_{0}+2 \sigma_{0}^{2}-2 \sigma_{1}+\pi_{0}\right)+\mu_{1} \pi_{1}+\left(1-y^{2}\right) \sigma_{0} \tau_{0}}{K_{0}^{2}\left(x^{2}-y^{2}\right)^{4}},
\end{aligned}
$$

where

$$
\begin{gathered}
\mu_{0}=-\frac{\hat{\rho}^{2}}{2 M^{2}}, \quad \sigma_{0}=-\frac{x^{2}-y^{2}}{2}+\beta_{0}\left(x^{2}+y^{2}\right)-2 \alpha_{0} x y, \\
\pi_{1}=4 x\left(\beta_{0} x-\alpha_{0} y\right)-\left(1+2 \beta_{0}\right)\left(x^{2}-y^{2}\right), \\
\mu_{1}=-\Delta_{0}\left(-1+x^{2}\right)^{2}+\frac{\sqrt{2} Q \beta_{0}^{2}}{\alpha_{0}}\left(x^{2}-y^{2}\right)^{2}, \\
\sigma_{1}=\Delta_{0}\left(x^{2}-y^{2}\right)+\left(-2 \Delta_{0} \beta_{0}+\beta_{1}\right)\left(x^{2}+y^{2}\right) \\
+2\left(2 \Delta_{0} \alpha_{0}-\alpha_{1}\right) x y,
\end{gathered}
$$

$$
\begin{gathered}
\pi_{0}=\left[1-4 \Delta_{0} \beta_{0}+\beta_{1}+\frac{\beta_{0}\left(\beta_{1}-K_{1}\right)}{K_{0}}\right] 4 x^{2} \\
-\sqrt{2}\left(P x\left(1+x^{2}\right)-Q y\left(1+y^{2}\right)\right) \\
+\left(\sqrt{2}\left(\beta_{0} P+\alpha_{0} Q\right) x-\sqrt{2}\left(\alpha_{0} P+\beta_{0} Q\right) y-\beta_{3}\right)\left(x^{2}-y^{2}\right) \\
+\left[4 \Delta_{0} \alpha_{0}-\alpha_{1}-\frac{\alpha_{0}\left(\beta_{1}-K_{1}\right)}{K_{0}}\right] 4 x y \\
\tau_{0}=\sqrt{2}(P x+Q y)\left(x^{2}-1\right)+\left(\alpha_{3}+\frac{Q}{\sqrt{2} \alpha_{0}} x\right)\left(x^{2}-y^{2}\right) \\
-\left(\alpha_{3}+\frac{Q}{\sqrt{2} \alpha_{0}}\right)\left(1-y^{2}\right),
\end{gathered}
$$

where we use prolate spheroidal coordinates

$$
\begin{aligned}
& x=\frac{\sqrt{\hat{\rho}^{2}+(\hat{z}+M)^{2}}+\sqrt{\hat{\rho}^{2}+(\hat{z}-M)^{2}}}{2 M}, \\
& y=\frac{\sqrt{\hat{\rho}^{2}+(\hat{z}+M)^{2}}-\sqrt{\hat{\rho}^{2}+(\hat{z}-M)^{2}}}{2 M},
\end{aligned}
$$

and introduce the notation

$$
\begin{gathered}
Q=\sqrt{1-P^{2}}, \quad \Delta_{0}=\frac{3-\sqrt{2} P}{2}, \quad K_{0}=\beta_{0}-1 / 2, \\
K_{1}=-\frac{1}{4}\left(\Delta_{0}-\frac{4}{\Delta_{0}}+7\right)\left(1-2 \beta_{0}\right)-\frac{2 \beta_{1} K_{0}}{1-2 \beta_{0}}-4 \Delta_{0} K_{0}, \\
\alpha_{0}=\frac{Q}{\sqrt{2}-2 P}, \quad \beta_{0}=-\sqrt{\alpha_{0}^{2}+1 / 2}
\end{gathered}
$$

$$
\begin{aligned}
& \alpha_{1}=\frac{2 Q^{2} \beta_{0}^{2}}{\alpha_{0}}-\frac{\Delta_{0}}{\sqrt{2}} Q\left(1+4 \beta_{0}^{2}\right), \\
& \beta_{1}=\frac{2 P Q \beta_{0}^{2}}{\alpha_{0}}-\frac{\Delta_{0}}{\sqrt{2}}\left(P+4 Q \alpha_{0} \beta_{0}\right),
\end{aligned}
$$

$\alpha_{3}=-\left(\frac{5 \Delta_{0}}{2}-\frac{2}{\Delta_{0}}+\frac{3}{2}\right)$,

$\beta_{3}=\frac{\Delta_{0}\left(1-8 \beta_{0}^{2}\right)+2\left(\alpha_{0} \alpha_{1}+\beta_{0} \beta_{1}\right)}{K_{0}}+\frac{\left(1-4 \beta_{0}^{2}\right) K_{1}}{2 K_{0}^{2}}$,

for $1 / \sqrt{2}<P \leq 1$. 


\section{B. Physical parameters}

Let us now consider the physical parameters of the generalized-NHEK2 solution. As we did at the level of the geometry, the near-horizon limiting procedure can be applied to the original physical parameters found in [22] (also reviewed here in Appendix A). Applying this technique yields the expressions for the masses $M_{1}, M_{2}$, and angular momenta $J_{1}, J_{2}$ in the generalized-NHEK2 solution

$M_{1}=\frac{M}{2}\left(1-\frac{Q}{\sqrt{2}-P}\right), \quad M_{2}=\frac{M}{2}\left(1+\frac{Q}{\sqrt{2}-P}\right)$,

$J_{1}=2 M_{1}^{2}\left(1-\frac{Q}{\sqrt{2}-P}\right)^{-1}, \quad J_{2}=2 M_{2}^{2}\left(1+\frac{Q}{\sqrt{2}-P}\right)^{-1}$,

and the corresponding angular velocities

$$
\Omega_{1}=\Omega_{2}=\frac{1}{2 M},
$$

satisfying at the same time the Smarr relation $M_{1}=2 J_{1} \Omega_{1}$ and $M_{2}=2 J_{2} \Omega_{2}$. It is worth noticing that the new solution contains objects that are in thermal equilibrium. The black hole entropy is, as usual, the area of the event horizon divided by 4 . This gives

$$
\begin{aligned}
& S_{1}=4 \pi M^{2}(2-\sqrt{2}(P+Q)), \\
& S_{2}=4 \pi M^{2}(2-\sqrt{2}(P-Q)) .
\end{aligned}
$$

\section{Ergospheres}

The generalized-NHEK2 spacetime that we constructed contains regions where the vector $\partial_{t}$ becomes null. We will refer to the boundary region as the ergosphere, since they are inherited from the presence of such regions in the original stationary extreme BBHs geometries. For NHEK2 these are defined by regions where $g_{t t}=0$ and give rise to a set of disconnected regions as shown in Fig. 1. Different values of the parameter $P$ are bounded by the extreme mass ratio solution when $P=1 / \sqrt{2}$ and identical mass solution when $P=1$. The horizons of the black holes in generalized-NHEK2 are points in the $(\hat{\rho}, \hat{z})$-plane and have finite horizon areas. There is a self-similar behavior close to each black hole that resembles the ergospheres' diagrams of isolated extremal Kerr black holes.

\section{Asymptotic behavior}

In the asymptotic limit, for $\hat{\rho}=r \sin \theta, \hat{z}=r \cos \theta$ and $r \rightarrow \infty$, the generalized-NHEK2 geometry in Sec. II A has a limiting metric that corresponds to the NHEK metric - in Weyl coordinates-(4) with functions

$f=\frac{4 M^{2} \hat{\rho}^{2}}{2 \hat{z}^{2}+\hat{\rho}^{2}}, \quad \omega=\frac{\sqrt{\hat{z}^{2}+\hat{\rho}^{2}}}{2 M^{2}}, \quad e^{2 \nu}=\frac{M^{2}\left(2 \hat{z}^{2}+\hat{\rho}^{2}\right)}{\left(\hat{z}^{2}+\hat{\rho}^{2}\right)^{2}}$.

In other words, the generalized-NHEK2 solution is asymptotically NHEK. It is worthwhile to mention at this point that in $[23,24]$ it was shown that in the case of 4D Einstein gravity the NHEK geometry is the unique (up to diffeomorphisms) regular stationary and axisymmetric solution asymptotic to NHEK with a smooth horizon. The NHEK2 geometry that we unveil is asymptotically NHEK, but is not
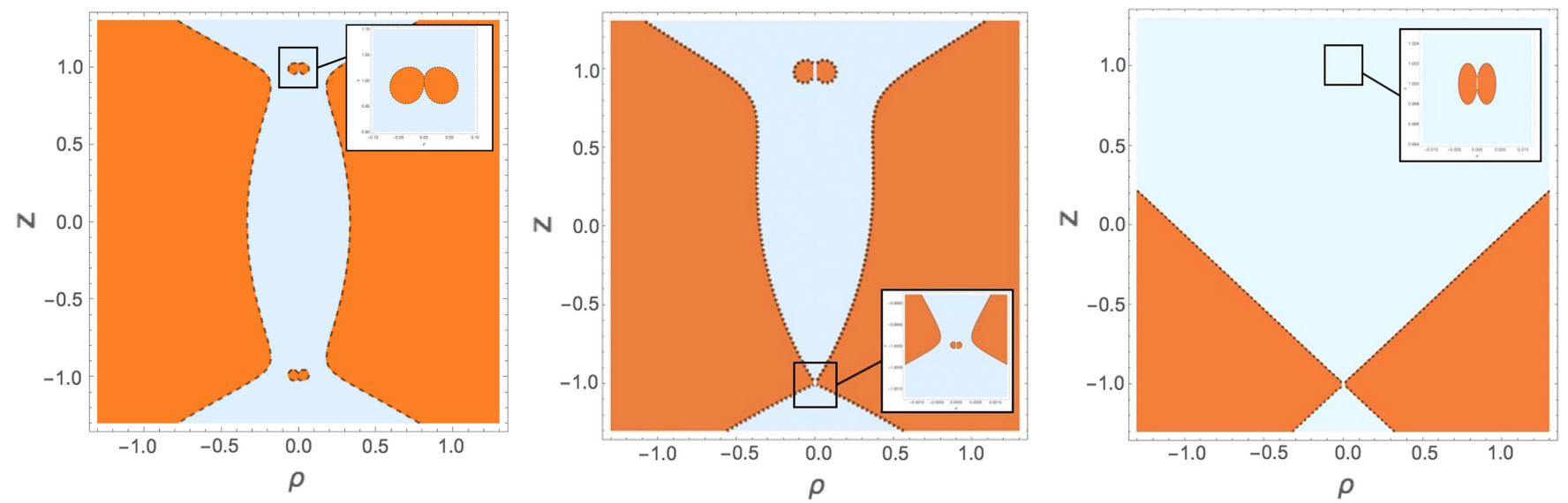

FIG. 1. Ergoregion (shaded orange region) of the generalized-NHEK2-black hole solution for $M=1$ and $P=1,0.9,0.71$ (from left to right). Each black hole is located at $\rho=\hat{\rho}_{H}=0, z=\hat{z}_{H}= \pm 1$. Magnified diagrams, close to the locations of the black holes appear in the corners. The dashed line corresponds to the boundary where $\partial_{\hat{t}}$ is null. The different values of $P$ in the diagrams correspond to solutions with BBHs of distinctive mass ratios. Note that in the generalized-NHEK2 solution the parameter $1 / \sqrt{2}<P \leq 1$ where $P=1$ is the equal mass identical black hole case, and $1 / \sqrt{2} \sim 0.07071$ the extreme mass ratio limit. 
diffeomorphic to NHEK; this is not in contradiction with the results of $[23,24]$ since the NHEK2 geometry is not smooth on the strut which keeps the BHs apart.

\section{E. Conical singularity}

As we have shown in the previous subsection, the generalized-NHEK2 is exactly asymptotically NHEK without any conical defects. However, as in the original stationary, extremal BBHs geometry there is in the bulk, a conical singularity on the $\hat{\rho}=0$ axis localized between the two black holes. In Weyl coordinates the conical singularities can be easily computed

$$
\Delta \hat{\phi}=2 \pi \lim _{\hat{\rho} \rightarrow 0}\left(1-\sqrt{\frac{f}{\hat{\rho}^{2} e^{2 \nu}}}\right), \quad-M<z<M .
$$

Our computation for the generalized-NHEK2 metric shows that there is a nonremovable conical excess between the two horizons.

$$
\Delta \hat{\phi}=2 \pi\left(1-\frac{1}{2(\sqrt{2}-P)^{2}}\right) .
$$

Outside this localized conical singularity our solutions are smooth.

\section{PIERCED-NHEK: NEAR HORIZON LIMIT AT FINITE SEPARATION}

In this section it is shown that there exists a well-defined near-horizon limit of the stationary binary extreme Kerr solution [21,22] even when the BHs, which are held apart by a conical singularity, are separated by a finite distance. The near-horizon region is composed of two disconnected NHEK-like geometries, one near each of the BHs. Each such geometry can be thought of as "NHEK pierced by a cosmic string," the strength of which is determined by the distance between the BHs. The cosmic string/conical singularity balances the gravitational attraction of the companion $\mathrm{BH}$, thereby enabling stationarity. The cosmic string extends all the way from the horizon to infinity in this geometry which we call the "pierced-NHEK."

Our starting point is the solution given in [21] (that corresponds to the identical mass binary black hole metric in [22] for $P=1$ which for convenience we reviewed in Appendix A). As the most general solution is quite involved, we will start by fixing the parameters at a specific, convenient value which will be enough to convey our point regarding the existence of a nonsingular nearhorizon geometry. It could be nice to explicitly write down the full most general expression, for arbitrary value of $P$, but for the sake of simplicity we will only focus on the $P=1$ case.

Starting with the solution presented in [21] with parameters $p=-1 / 2, \quad \kappa=4(\sqrt{33}-1)^{-1}$ (which sets $M=1, J=2$ ) and coordinates denoted by $\{\rho, z, t, \phi\}$, we choose to focus on the $\mathrm{BH}$ located at $\tilde{z}=\kappa$ and use the transformation

$$
\rho=\epsilon R \sin \Theta, \quad z-\kappa=\epsilon R \cos \Theta
$$

$t=\frac{17+\sqrt{33}}{16} \frac{T}{\epsilon}, \quad \phi=\left(\Phi+\omega_{0} \frac{17+\sqrt{33}}{16} \frac{T}{\epsilon}\right)$,

where $\omega_{0}=(\sqrt{11}-\sqrt{3}) / 4$ facilitates the transition into a frame which corotates with the BH. Taking $\epsilon \rightarrow 0$ yields the nonsingular geometry

$$
\begin{aligned}
d s^{2}= & \Gamma(\Theta)\left[-R^{2} d T^{2}+\frac{d R^{2}}{R^{2}}+d \Theta^{2}\right. \\
& \left.+\Lambda^{2}(\Theta)\left(d \Phi+\frac{\sqrt{11}-\sqrt{3}}{2} R d T\right)^{2}\right],
\end{aligned}
$$

where

$$
\begin{gathered}
\Gamma(\Theta)=\frac{2(3 \sqrt{33}-13) \cos \Theta+(15-\sqrt{33})(3+\cos 2 \Theta)}{16}, \\
\Gamma(\Theta) \Lambda(\Theta)^{2}=\frac{256 \sin ^{2} \Theta}{4(-59+11 \sqrt{33}) \cos \Theta+(93-13 \sqrt{33})(3+\cos 2 \Theta)} .
\end{gathered}
$$

In this geometry, a priori, there could be a conical singularity either at $\Theta=0$ or at $\Theta=\pi$. Using (27), however, shows explicitly that at $\Theta=0$ there is no conical singularity whereas for $\theta=\pi$ there is an angular excess of

$$
\Delta \Phi=2 \pi \frac{\sqrt{33}-1}{8}
$$

Writing the pierced-NHEK geometry in the form (30) shows immediately that it enjoys the isometry group $\mathrm{SL}(2, \mathrm{R}) \times \mathrm{U}(1)$, just like NHEK: the strut on the symmetry axis does not spoil this symmetry. 


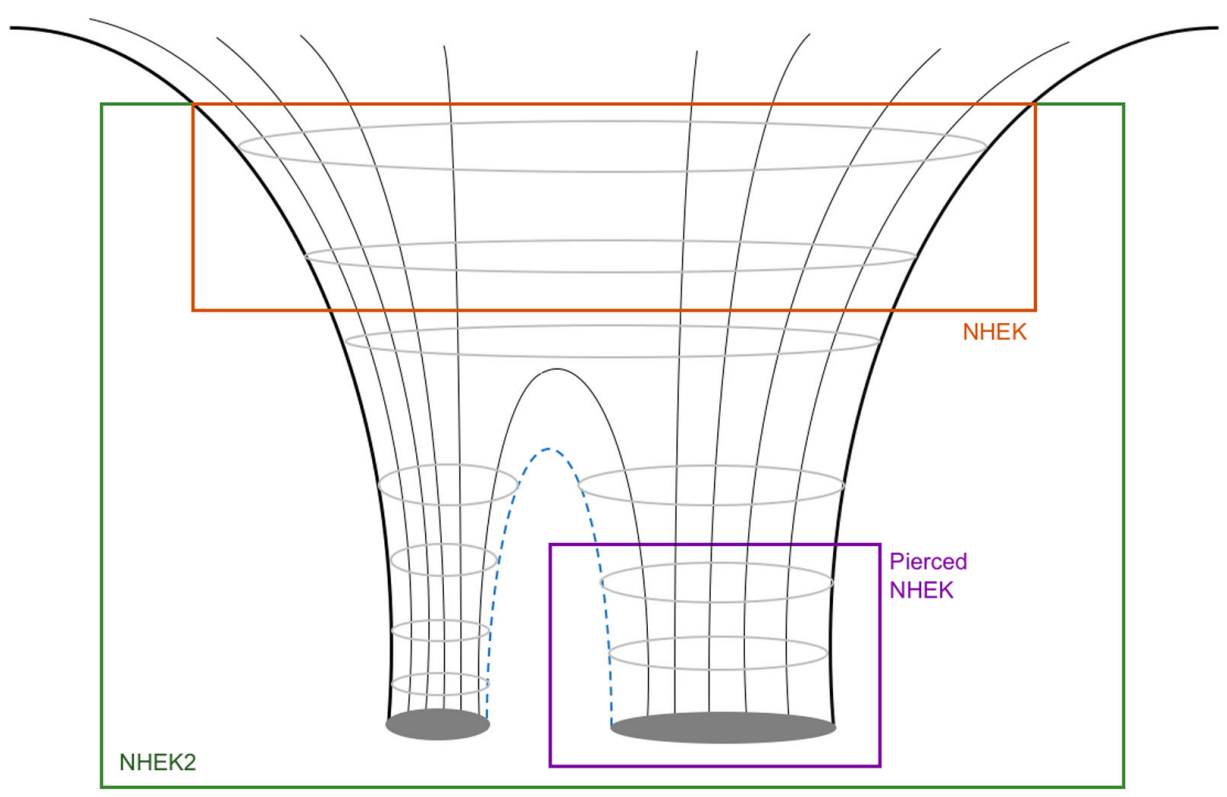

FIG. 2. The diagram represents a spatial cross section of the two extremal corotating BHs metric [22] reproduced in Appendix A. The geometry (in black and gray) has an asymptotically Minkowskian region and a single black hole throat of mass $M_{1}+M_{2}$ which divides into two throats of masses $M_{1}$ and $M_{2}$. The strut (conical singularity) in the solution is localized between the two black holes (dashed blue). In the infrared limit $\lambda \rightarrow 0$, when zooming into the near horizon limit, the throat becomes infinitely long and the Minkowski region decouples. This is the new generalized NHEK2 solution that we constructed (represented in green) that is asymptotically NHEK (in orange). The splitting of the throat into two pieces survives this limit. In the deep infrared when zooming close to one of the horizons, we find new geometries that we call "pierced NHEK." These correspond to the NHEK metric pierced by a conical singularity on the symmetry axis, which runs from one of the poles up to the boundary (in purple).

\section{DISCUSSION}

The aim of this paper was to unveil and analyze the generalized-NHEK2 geometry. This geometry is obtained via a limiting procedure that we developed: a zoom-in on the near-horizon region of a 1-parameter family of corotating, ${ }^{2}$ double-extreme Kerr solutions of arbitrary masses where the two BHs are parametrically close to each other and are held apart by a conical singularity (strut). The distance between the BHs is scaled to zero at the same rate of the zoom-in on the near-horizon region. This gives a relatively simple solution, which is asymptotically NHEK, and in the infrared flows to two separate throats which we call "pierced-NHEK" geometries: each of them is, approximately (when zooming further towards one of the horizons), NHEK pierced by a conical singularity on the symmetry axis, which runs from one of the poles up to the boundary. We find that in the deep infrared where the geometry is approximately pierced-NHEK, the presence of the strut does not break the isometry group $\operatorname{SL}(2, R) \times$ $\mathrm{U}(1)$ - it is restored there. In Fig. 2, we illustrate the structure of the generalized-NHEK2 geometry. The generalized-NHEK2 solution asymptotes to NHEK, yet it is

\footnotetext{
${ }^{2}$ The counterrotating counterpart cannot be used to construct a similar solution since asymptotically it appears as a nonextreme $\mathrm{BH}$
}

not diffeomorphic to NHEK. This is not in contradiction to the discussions in $[25,26]$ since in these papers, smoothness is assumed while here we allow for a conical singularity which balances the gravitational attraction between the BHs. This paper generalizes the construction studied recently in [11] for the equal mass case.

\section{ACKNOWLEDGMENTS}

We would like to thank David Chow and Oscar Varela for helpful discussions. This work was supported by the NSF Grant No. PHY-1707571 at Utah State University and, partially, by the Max Planck Gesellschaft through the Gravitation and Black Hole Theory Independent Research Group. S. H. gratefully acknowledges support by the Jacob Goldfield Postdoctoral Support Fund.

\section{APPENDIX: STATIONARY EXTREME KERR BINARY WITH STRUT-FULL SOLUTION}

Here we record, for completeness, the full exact solution corresponding to two extremal co-rotating $\mathrm{BHs}$ which are held apart by a strut lying on the joint rotation axis, between the BHs. The solution is axisymmetric, stationary and asymptotically flat. We follow the conventions of [22] in which this solution was presented. 
Define prolate spheroidal coordinates $(x, y)$ by

$$
\begin{aligned}
& x=\frac{\sqrt{\rho^{2}+(z+\kappa)^{2}}+\sqrt{\rho^{2}+(z-\kappa)^{2}}}{2 \kappa}, \\
& y=\frac{\sqrt{\rho^{2}+(z+\kappa)^{2}}-\sqrt{\rho^{2}+(z-\kappa)^{2}}}{2 \kappa},
\end{aligned}
$$

the metric is given by

where:

$$
\begin{aligned}
f= & \frac{\kappa\left(y^{2}-1\right) F}{D \omega}, \quad e^{2 \nu}=\frac{D}{K_{0}^{2}\left(x^{2}-y^{2}\right)^{4}}, \quad \omega=-\frac{\kappa\left(y^{2}-1\right) F N}{\left[\left(\kappa\left(y^{2}-1\right) F\right)^{2}-\rho^{2} D^{2}\right]}, \\
N= & \mu^{2}-\left(x^{2}-1\right)\left(1-y^{2}\right) \sigma^{2}, \\
D= & N+\mu \pi+\left(1-y^{2}\right) \sigma \tau, \\
F= & \left(x^{2}-1\right) \sigma \pi+\mu \tau, \\
\mu= & p^{2}\left(p^{2}\left(x^{2}-1\right)^{2}+q^{2}\left(1-y^{2}\right)^{2}+\left(\alpha^{2}-\beta^{2}\right)\left(x^{2}-y^{2}\right)^{2}\right), \\
\sigma= & p^{2}\left(2\left[p q\left(x^{2}-y^{2}\right)+\beta\left(x^{2}+y^{2}\right)-2 \alpha x y\right]\right), \\
\pi= & p^{2}\left(( 4 p ^ { 2 } / K _ { 0 } ) \left\{\left(K_{0} / p^{2}\right)\left[p P s x\left(x^{2}+1\right)+2 x^{2}+q Q y\left(y^{2}+1\right)\right]\right.\right. \\
& +2(p Q+p P \alpha+q Q \beta)\left[p q y\left(x^{2}-y^{2}\right)+\beta y\left(x^{2}+y^{2}\right)-2 \alpha x y^{2}\right] \\
& -\left(K_{0} / p^{2}\right)\left(x^{2}-y^{2}\right)[(p Q \alpha-q P \beta) x+(q P \alpha-p Q \beta) y]-2\left(q^{2} \alpha^{2}+p^{2} \beta^{2}\right)\left(x^{2}-y^{2}\right) \\
& \left.\left.+4(p q+\beta)\left(\beta x^{2}-\alpha x y\right)\right\}\right), \\
\tau= & p^{2}\left(( 4 p ^ { 2 } / K _ { 0 } ) \left\{\left(K_{0} / p^{2}\right) x\left[(q Q \alpha+p P \beta)\left(x^{2}-y^{2}\right)-q P\left(1-y^{2}\right)\right]\right.\right. \\
& +(p Q+p P \alpha+q Q \beta) y\left[\left(p^{2}-\alpha^{2}+\beta^{2}\right)\left(x^{2}-y^{2}\right)+y^{2}-1\right] \\
& \left.\left.-p Q\left(K_{0} / p^{2}\right) y\left(x^{2}-1\right)-2 p\left(q \alpha^{2}-q \beta^{2}-p \beta\right)\left(x^{2}-y^{2}\right)-(p q+\beta)\left(1-y^{2}\right)\right\}\right), \\
K_{0}= & p^{2}\left(p^{2}+\alpha^{2}-\beta^{2}\right),
\end{aligned}
$$

and the parameters are constrained so that

$$
p^{2}+q^{2}=1, \quad P^{2}+Q^{2}=1 .
$$

For the corotating solution in which we are interested in this paper,

$$
\begin{aligned}
& \alpha=-\frac{Q\left[q \Delta+p q^{2}+P\left(1+p^{2}\right)\right]}{2\left(p^{2}-Q^{2}\right)}, \\
& \beta=\frac{p\left[P \Delta+q\left(1+p P+Q^{2}\right)\right]}{2\left(p^{2}-Q^{2}\right)}, \\
& \Delta=\sqrt{4 p^{2}(1+p P)+q^{2}(p+P)^{2}} .
\end{aligned}
$$

\section{Physical parameters}

The asymptotic metric does not contain a conical singularity, then the mass $M_{1}, M_{2}$ and the angular momenta $J_{1}, J_{2}$ of the black holes can be easily calculated

$$
\begin{aligned}
& M_{1}=\frac{\kappa\left[\left(q+p q P-p^{2} Q\right) \Delta-(1+p P)\left(p+p^{3}+q^{2} P-p q Q\right)+p q^{3} Q\right]}{2 p(1+p P)\left(p^{2}-q^{2}\right)}, \\
& M_{2}=\frac{\kappa\left[\left(q+p q P+p^{2} Q\right) \Delta-(1+p P)\left(p+p^{3}+q^{2} P+p q Q\right)-p q^{3} Q\right]}{2 p(1+p P)\left(p^{2}-q^{2}\right)},
\end{aligned}
$$




$$
\begin{aligned}
& J_{1}=\frac{(1+p P+q Q) M_{1}^{2}}{2(p+P)^{2}}\left[\left(1+p P+q^{2}\right) \Delta-4 p q+p q(p-P)^{2}\right], \\
& J_{2}=\frac{(1+p P-q Q) M_{2}^{2}}{2(p+P)^{2}}\left[\left(1+p P+q^{2}\right) \Delta-4 p q+p q(p-P)^{2}\right],
\end{aligned}
$$

and, employing the Smarr relation, we can easily find the expressions for the angular velocities

$$
\Omega_{1}=\frac{M_{1}}{2 J_{1}}, \quad \Omega_{2}=\frac{M_{2}}{2 J_{2}} .
$$

Additionally, the entropy for each black hole can be calculated to give:

$$
\begin{aligned}
& S_{1}=\frac{2 \pi p^{2} \kappa^{2}}{K_{0}^{2}}\left(K_{0}(1+p P+q Q)-2 p^{2}(\alpha-\beta)(p(Q+P \alpha+q)+(q Q+1) \beta)\right), \\
& S_{2}=\frac{2 \pi p^{2} \kappa^{2}}{K_{0}^{2}}\left(K_{0}(1+p P-q Q)-2 p^{2}(\alpha+\beta)(p(Q+P \alpha-q)+(q Q-1) \beta)\right) .
\end{aligned}
$$

[1] L. Gou, J. E. McClintock, M. J. Reid, J. A. Orosz, J. F. Steiner, R. Narayan, J. Xiang, R. A. Remillard, K. A. Arnaud, and S. W. Davis, The extreme spin of the black hole in Cygnus x-1, Astrophys. J. 742, 85 (2011).

[2] G. Risaliti et al., A rapidly spinning supermassive black hole at the centre of NGC 1365, Nature (London) 494, 449 (2013).

[3] A. C. Fabian, The innermost extremes of black hole accretion, Astron. Nachr. 337, 375 (2016).

[4] J. E. McClintock, R. Shafee, R. Narayan, R. A. Remillard, S. W. Davis, and L. x. Li, The spin of the near-extreme Kerr black hole GRS $1915+105$, Astrophys. J. 652, 518 (2006).

[5] K. Akiyama et al. (Event Horizon Telescope Collaboration), First M87 event horizon telescope results. I. The shadow of the supermassive black hole, Astrophys. J. 875, L1 (2019).

[6] J. M. Bardeen and G. T. Horowitz, The extreme Kerr throat geometry: A vacuum analog of $\mathrm{AdS}_{2} \times \mathrm{S}^{2}$, Phys. Rev. D 60 , 104030 (1999).

[7] M. Guica, T. Hartman, W. Song, and A. Strominger, The Kerr/CFT correspondence, Phys. Rev. D 80, 124008 (2009).

[8] J. B. Hartle and S. W. Hawking, Solutions of the EinsteinMaxwell equations with many black holes, Commun. Math. Phys. 26, 87 (1972).

[9] J. M. Maldacena, J. Michelson, and A. Strominger, Anti-de Sitter fragmentation, J. High Energy Phys. 02 (1999) 011.

[10] G. W. Gibbons and C. M. Hull, A bogomolny bound for general relativity and solitons in $N=2$ supergravity, Phys. Lett. 109B, 190 (1982).
[11] J. Ciafre and M. J. Rodriguez, A near horizon extreme binary black hole geometry, arxiv:1804.06985.

[12] J. Camps, S. Hadar, and N. S. Manton, Exact gravitational Wwave signatures from colliding extreme black holes, Phys. Rev. D 96, 061501 (2017).

[13] P. V. P. Cunha, C. A. R. Herdeiro, and M. J. Rodriguez, Does the black hole shadow probe the event horizon geometry?, Phys. Rev. D 97, 084020 (2018).

[14] A. Anabalon, M. Appels, R. Gregory, D. Kubiznk, R. B. Mann, and A. Ovgun, Holographic thermodynamics of accelerating black holes, Phys. Rev. D 98, 104038 (2018).

[15] W. Kinnersley and D. M. Chitre, Symmetries of the stationary EinsteinMaxwell equations. IV. Transformations which preserve asymptotic flatness, J. Math. Phys. (N.Y.) 19, 2037 (1978).

[16] D. Kramer and G. Neugebauer, The superposition of two Kerr solutions, Phys. Lett. 75A, 259 (1980).

[17] C. A. R. Herdeiro and C. Rebelo, On the interaction between two Kerr black holes, J. High Energy Phys. 10 (2008) 017.

[18] V.S. Manko, E. D. Rodchenko, E. Ruiz, and B. I. Sadovnikov, Exact solutions for a system of two counter-rotating black holes, Phys. Rev. D 78, 124014 (2008).

[19] M. S. Costa, C. A. R. Herdeiro, and C. Rebelo, Dynamical and thermodynamical aspects of interacting Kerr black holes, Phys. Rev. D 79, 123508 (2009).

[20] V.S. Manko, E. D. Rodchenko, E. Ruiz, and B. I. Sadovnikov, On the simplest binary system of rotating black holes, AIP Conf. Proc. 1122, 332 (2009). 
[21] V. S. Manko and E. Ruiz, On a simple representation of the Kinnersley-Chitre metric, Prog. Theor. Phys. 125, 1241 (2011).

[22] V. S. Manko, E. Ruiz, and M. B. Sadovnikova, Stationary configurations of two extreme black holes obtainable from the Kinnersley-Chitre solution, Phys. Rev. D 84, 064005 (2011).

[23] A. J. Amsel, G. T. Horowitz, D. Marolf, and M. M. Roberts, No dynamics in the extremal Kerr throat, J. High Energy Phys. 09 (2009) 044.
[24] O. J. C. Dias, H. S. Reall, and J.E. Santos, Kerr-CFT and gravitational perturbations, J. High Energy Phys. 08 (2009) 101.

[25] A. J. Amsel, G. T. Horowitz, D. Marolf, and M. M. Roberts, Uniqueness of extremal Kerr and Kerr-Newman black holes, Phys. Rev. D 81, 024033 (2010).

[26] H. K. Kunduri and J. Lucietti, Classification of near-horizon geometries of extremal black holes, Living Rev. Relativity 16, 8 (2013). 